\title{
On using textural information from longitudinal PET scans for predicting tumor response
}

Jose George ${ }^{1}$, Kathleen Vunckx ${ }^{2}$, Sabine Tejpar ${ }^{3}$, Christophe M. Deroose ${ }^{2}$, Johan Nuyts ${ }^{2}$, Dirk Loeckx ${ }^{1}$ and Paul Suetens ${ }^{1}$

${ }^{1}$ Center for Processing Speech and Images, Department of Electrical Engineering, KU Leuven, Leuven, Belgium

${ }^{2}$ Department of Nuclear Medicine, University Hospital Leuven, Leuven, Belgium

${ }^{3}$ Department of Gastroenterology, University Hospital Leuven, Leuven, Belgium

${ }^{4}$ icometrix NV, Leuven, Belgium

${ }^{5}$ IBBT-K.U.Leuven Future Health Department, Belgium

*Corresponding author:

Jose George, PhD Student,

Medical Imaging Research Center, University Hospital Gasthuisberg,

Herestraat 49 - bus 7003, B-3000 Leuven, Belgium

E-mail: jose.george@uzleuven.be Tel: +32(0) 16-34.90.41 / Fax: +32(0) 16-34.90.01

Words: 570

Financial support: This work is financially supported by K.U.Leuven's Concerted Research Action GOA/11/006, Research Foundation - Flanders (FWO) and IWT Agency for Innovation by Science and Technology - Applied Biomedical Research (TBM) project 070717.

Running Title: Therapy response from tumor texture 
Purpose: Early therapy response prediction, employing biomarkers such as [F-18]fluorodeoxyglucose (FDG) followed with positron emission tomography (PET) for imaging the tumor metabolic activity, is a very actively researched topic. Traditionally, only the first order intensity based feature estimates extracted from the longitudinal scans are used for the response evaluations. Nonetheless, lesion heterogeneity computed from the higher order textures of the baseline scan prior to treatment has been recently found to be correlating well with the final outcome. The aim of this study is to establish the significance of tumor texture in follow up evaluations.

Methods: 15 patients suffering from chemo refractory metastatic colorectal cancer and treated with cetuximab were selected for the study. Baseline FDG PET scans were acquired prior to treatment and the follow up scanned at 1 week after $1^{\text {st }}$ dose of treatment was investigated for early response. One to five lesions were identified per patient. Intensity based features (IF)

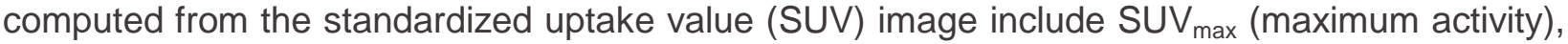
$S_{\text {SUean }}$ (mean activity), SUV peak (highest mean activity in $1 \mathrm{~mL}$ sphere within tumor), tumor lesion volume (TLV) in $\mathrm{mL}$, total lesion activity (TLA), TLA $=\mathrm{SUV}_{\text {mean }} \times \mathrm{TLV}$ in SUVmL and total lesion energy (TLE), TLE $=\operatorname{SUV}_{\text {mean }}{ }^{\wedge} 2 \times \mathrm{TLV}$ in SUV^2mL. Texture covariates (TF) estimated include 11 activity level run length matrix (ALRL) features, 11 activity level size zone matrix (ALSZ) features, 7 activity level co occurrence matrix (ALCM) features, 5 activity level difference matrix (ALDC) features and 19 activity level histogram (ALHS) features. Response indices (RI) were computed as the \% change with respect to baseline after adding up covariates over all lesions per patient under study. The dynamic range of IF was investigated in baseline. A Cox proportional hazards regression model was fit with Rls and the time dependent receiver operating characteristics (ROC) was determined. Leave one-out cross validation (LOCV) was performed to investigate the fitting of the model. The concordance measure with the time to progression (TTP) was determined in each of the scenarios.

Results: The baseline IF dynamic ranges are listed below representing (Median \pm Standard Deviation, Minimum - Maximum) the respective covariates. SUV $\max (6.33 \pm 2.34,2.40-12.28)$. $\operatorname{SUV}_{\text {peak }}(4.86 \pm 2.01,1.64$ - 10.52). SUV mean $(4.05 \pm 1.52,1.61-8.39) . \operatorname{TLV}(5.71 \pm 155.91,0.48-$ 1182.42). TLA (29.58 \pm 872.80, 2.11 - 6620.88). TLE (152.79 \pm 5138.32, 3.55 - 38963.30). Cox model on LOCV with the IF only covariate set and the IF+TF set resulted in concordance of 0.6570 and 0.6764 resp.

Conclusion: The results with the current data denote an added value in using textural information for therapy response evaluation. Moreover, therapy response could be predicted as early as 1 week after $1^{\text {st }}$ dose of treatment from the feature estimates. 